\title{
Diaphragmatic and Genioglossus Electromyographic Activity at the Onset and at the End of Obstructive Apnea in Children with Obstructive Sleep Apnea Syndrome ${ }^{1}$
}

\author{
J. P. PRAud, A. M. D'Allest, M. F. Delaperche, S. Bobin, AND Cl. Gaultier \\ Laboratory of Physiology, CNRS UA 1159 and ENT Department [S.B.], Hospital A. Béclère, Clamart, France
}

\begin{abstract}
We studied the activity of the diaphragm and of the genioglossus at the onset and at the end of obstructive sleep apnea in children. Seven children (mean age 46 months, range 15-87) with obstructive sleep apneas mainly due to enlarged tonsils were tested during natural sleep. We recorded sleep stages (neurophysiological criteria), nasal and buccal air flow (thermistors), thoracoabdominal motion (magnetometers), genioglossus and diaphragm electromyographic activity (EMG) (surface electrodes), and transcutaneous partial pressure of oxygen (Radiometer $44^{\circ} \mathrm{C}$ ). A total of 153 obstructive apneas for the whole group of patients was studied. Compared to the preceding unoccluded breaths, genioglossus and diaphragm EMG data showed that 1) at the onset of obstructive apnea there was no significant decrease in genioglossus and/or diaphragm EMG, contrasting with published data for obese adults, and 2) at the end of obstructive apnea, significant preferential increase in genioglossus EMG, not related to the decrease in transcutaneous partial pressure of oxygen, was found as in obese adults. This study showed that different mechanisms may control the onset of obstructive apnea in children as compared to adults, whereas children and obese adults share the same preferential increase in genioglossus EMG at the end of obstructive apnea. (Pediatr Res 23: 1-4, 1988
\end{abstract}

\section{Abbreviations}

OSAS, obstructive sleep apnea syndrome EMG, electromyographic activity

$\mathrm{TcPO}_{2}$, transcutaneous partial pressure of oxygen

NREM, nonrapid eye movement sleep

REM, rapid eye movement sleep

Pathogenesis of the OSAS in obese adults has yet to be entirely defined. Current concepts of upper airway patency emphasize the role of the critical balance between upper airway dilating muscles, especially the genioglossus, and inspiratory muscles, especially the diaphragm (1). Previous studies in obese adults have suggested that several different mechanisms may be involved at the onset of obstructive apnea, i.e. abnormal function of upper airway dilating muscles $(1,2)$ or decrease in central

Received February 20, 1987; accepted August 17, 1987

Correspondence and reprint requests J. P. Praud, Laboratory of Physiology, Hospital Antoine Beclere, 9214l Clamart, France.

Supported by grants from the Faculty of Medecine Paris XI and CNRS.

${ }^{1}$ Presented in part at the annual meeting of the American Thoracic Society, Kansas City, MO, 1986 neural drive to both upper airway and inspiratory muscles (3). There is a consensus as to the mechanism that explains the end of obstructive apnea in obese adults; authors agree that the essential factor is preferential activation of upper airway dilating muscles $(1,3)$.

In infants and children with OSAS few studies have concerned genioglossus and/or diaphragm EMG activity. In children studied during afternoon naps with enlarged tonsils, Jeffries et al. (4) reported that the genioglossus muscle was recruited in contrast to children without obstructive sleep apneas. Similarly, in four micrognathic infants during night sleep, Roberts et al. (5) observed recruitment of the genioglossus. However, in these previous reports no attempt was made to study the EMG activity of both genioglossus and diaphragm at the onset and at the end of obstructive apnea. Thus the present study was designed to see whether or not upper airway and inspiratory muscle behavior at the onset and at the end of obstructive apnea in children with OSAS are similar to those seen in adults.

\section{MATERIALS AND METHODS}

Seven nonobese children with clinical symptoms of upper airway obstruction were studied (Table 1). The causes were enlarged tonsils and/or adenoids in six children associated with Crouzon disease in patient 2 and with Down's syndrome in patient 3. Patient 4 had temporomandibular joint ankylosis. Parental consent was obtained for the studies.

Sleep studies were conducted under the surveillance of a physician during an afternoon nap (patients 5 and 7) or night sleep (patients 1 to 4 ). No sedation nor sleep deprivation were used, because of their depressive effect on genioglossus activity $(6,7)$. Sleep stages were scored according to neurophysiological criteria (8), from electroencephalography, submental electromyogram, and electrooculography. The following respiratory parameters were recorded: 1) nasal and oral airflow (thermistors), 2) rib cage and abdominal anteroposterior diameters (magnetometers on the nipple line and just above the umbilicus), 3) $\mathrm{TcPO}_{2}$ (Radiometer electrode heated to $44^{\circ} \mathrm{C}$ ). Surface electrodes were used to record both diaphragmatic (9-11) and genioglossus EMG $(4,12,13)$. For the diaphragm, two silver cup electrodes $(d=5$ $\mathrm{mm}$ ) were placed in the 8th right intercostal space, on the anterior and midaxillary lines, since preliminary personal observations using subcostal locations showed that the largest phasic inspiratory activity with the smallest electrocardiographic signal could be recorded in the eighth right intercostal space. For the genioglossus, cups were placed on the midline; one just under the lower lip, the other between the chin and hyoid bone, as previously described in infants (12) and adults (13). Cups were filled with electrode jelly and secured with adhesive tape. EMGs were amplified and filtered (Electromed). All the above described 
parameters were simultaneously displayed on a 16 channel recorder (Alvar) $(20 \mathrm{~mm} / \mathrm{s})$.

\section{RESULTS}

Apneas (defined as total absence of air flow during at least 5 s) were counted and classified as central or obstructive. In addition, hypopneas (decrease in inspiratory air flow of more than $50 \%$ during at least $5 \mathrm{~s}$ ) were also considered for calculation of the apnea index, which is the number of apneas and hypopneas per hour of sleep (14). Results of the apnea index for each patient are listed in Table 1.

Genioglossus and diaphragmatic EMG activity were quantified as previously described in children by Jeffries et al. (4) by measuring maximal amplitude from peak to peak during inspiratory bursts. Genioglossus and diaphragmatic EMG activities at the onset and at the end of obstructive apnea were expressed as a percentage of the control values calculated in the same way from the three preceding unoccluded breaths. In our group of patients, 153 obstructive apneas were used for the EMG study at the onset and at the end of apnea. For each patient the duration and the repartition according to sleep stage of the obstructive apneas are listed in Table 2. Because of the rapid succession of apneas in 38 of the 153 apneas studied, calculation of the control values in these cases was made from three breaths taken from the same sleep stage during the preceding minutes.

Statistical comparison between diaphragmatic and genioglossus EMG control values and values at the onset and at the end of the obstructive apnea was made using the nonparametric Wilcoxon test for paired data (15).

\section{RESULTS}

Figure 1 shows an example of diaphragmatic and genioglossus EMG recording in patient 2 during the control unoccluded breaths and during an obstructive apnea. During the unoccluded breaths phasic inspiratory EMG of both muscles was present.

Mean changes in diaphragmatic and genioglossus EMG for each patient [indicated by his corresponding symbol (Table 1)], expressed as a percentage of the preceding unoccluded breaths, are reported in Figure 2 for the onset of obstructive apnea and in Figure 3 for the end of obstructive apnea.

At the onset of obstructive apnea (Fig. 2) in both sleep stages in five patients (nos. 1-5) diaphragmatic and genioglossus EMG were lower than in the control unoccluded breaths. In patients 6 and 7 diaphragmatic and genioglossus EMG were equal to control unoccluded breaths. However, for the group as a whole, diaphragmatic EMG (mean $\pm 1 \mathrm{SD}, 86 \% \pm 11$ ) and genioglossus EMG (mean $\pm 1 \mathrm{SD}, 82 \% \pm 14$ ) were not significantly different from control values. Moreover the change in genioglossus EMG was not significantly different from the change in diaphragmatic EMG.
At the end of obstructive apnea (Fig. 3), when compared to control unoccluded breaths, in either sleep stage, genioglossus EMG increased in all subjects (mean $\pm \mathrm{SD}, 157 \% \pm 21$ ). Increase in genioglossus EMG was significant for the group as a whole ( $p$ $<0.01)$. Increase in diaphragmatic EMG was present in all subjects and significant $(p<0.01)$ only in NREM sleep; in REM sleep, diaphragmatic EMG increased in four cases (patients 1, 2, 3 , and 4) but was lower than during unoccluded breaths in patients 5 and 6 . Finally, genioglossus EMG increased significantly more than diaphragmatic EMG (mean \pm SD, $108 \% \pm 45$ ) $(p<0.01)$ when both NREM and REM sleep were taken into account.

In 33 obstructive apneas where comparison of increase in genioglossus EMG and decrease in $\mathrm{TcPO}_{2}$ induced by apnea was available no significant relationship was found.

\section{DISCUSSION}

We studied diaphragmatic and genioglossus EMG during the course of obstructive apnea in seven unsedated, nonsleep deprived children with OSAS in order to elucidate the pathogenesis of obstructive sleep apnea in children. Results show that 1) at the onset of obstructive apnea, diaphragmatic and genioglossus EMG, when compared to the preceding unoccluded breaths, did not significantly change and 2) at the end of obstructive apnea, genioglossus EMG significantly increased, this increase being significantly greater than the increase in diaphragmatic EMG and not related to the decrease in $\mathrm{TcPO}_{2}$ induced by obstructive apnea.

Table 2. Duration and repartition according to sleep state of 153 studied obstructive apneas in seven patients

\begin{tabular}{|c|c|c|c|}
\hline \multirow[b]{2}{*}{ Patient } & \multirow{2}{*}{$\begin{array}{l}\text { Mean duration } \\
\text { (s) }\end{array}$} & \multicolumn{2}{|c|}{ Repartition } \\
\hline & & NREM sleep & REM sleep \\
\hline 1 & $\begin{array}{l}8.5 \pm 3^{*} \\
(5-18) \dagger\end{array}$ & 2 & 19 \\
\hline 2 & $\begin{array}{c}14.4 \pm 4.5 \\
(5-23)\end{array}$ & 11 & 15 \\
\hline 3 & $\begin{array}{l}8.5 \pm 3 \\
(5-17)\end{array}$ & 7 & 16 \\
\hline 4 & $\begin{array}{c}14.5 \pm 7.5 \\
(5-31)\end{array}$ & 11 & 18 \\
\hline 5 & $\begin{array}{l}9 \pm 3.5 \\
(5-18)\end{array}$ & 13 & 15 \\
\hline 6 & $\begin{array}{l}5 \pm 0.5 \\
(5-6)\end{array}$ & & 5 \\
\hline 7 & $\begin{array}{l}10 \pm 3 \\
(6-15)\end{array}$ & 21 & \\
\hline
\end{tabular}

$* \pm 1 \mathrm{SD}$

$\dagger$ Range.

Table 1. Etiology and characteristics of sleep obstructive syndrome in seven studied children

\begin{tabular}{|c|c|c|c|c|c|c|c|c|}
\hline Patient & Symbol & Sex & $\begin{array}{l}\text { Age } \\
(\mathrm{mo})\end{array}$ & Etiology & $\begin{array}{l}\text { Total sleep } \\
\text { time }(\mathrm{mn})\end{array}$ & $\begin{array}{l}\% \text { of REM } \\
\text { sleep }\end{array}$ & $\begin{array}{l}\text { Index of } \\
\text { apneas }\end{array}$ & $\begin{array}{c}\% \text { obstructive } \\
\text { A/H }\end{array}$ \\
\hline 1 & $*$ & $M$ & 18 & $\mathrm{ET}+\mathrm{EA}$ & 400 & 28 & 24 & 79 \\
\hline 2 & $\square$ & $\mathrm{F}$ & 55 & $\begin{array}{l}\text { ET + Crouzon dis- } \\
\quad \text { ease }\end{array}$ & 530 & 15 & 41 & 87 \\
\hline 3 & $\bullet$ & M & 76 & $\begin{array}{l}\text { ET + Down syn- } \\
\text { drome }\end{array}$ & 480 & 30 & 22 & 69 \\
\hline 4 & $\Delta$ & F & 87 & $\begin{array}{l}\text { Temporomandibular } \\
\text { joint ankylosis }\end{array}$ & 460 & 11 & 15 & 97 \\
\hline 6 & $\square$ & M & 15 & EA & 95 & 18 & 13 & 86 \\
\hline 7 & $\Delta$ & M & 20 & ET & 115 & 12 & 28 & 89 \\
\hline
\end{tabular}

M, male; F, female; ET, enlarged tonsils; EA, enlarged adenoids; A/H, apneas and hypopneas longer than $5 \mathrm{~s}$; index of apneas, number of A/H per sleep hour; \% obstructive $\mathrm{A} / \mathrm{H}$, obstructive $\mathrm{A} / \mathrm{H}$ as a percentage of total number of apneas. Symbol noticed for each patient was used to plot individual data in Figures 1 and 2. 


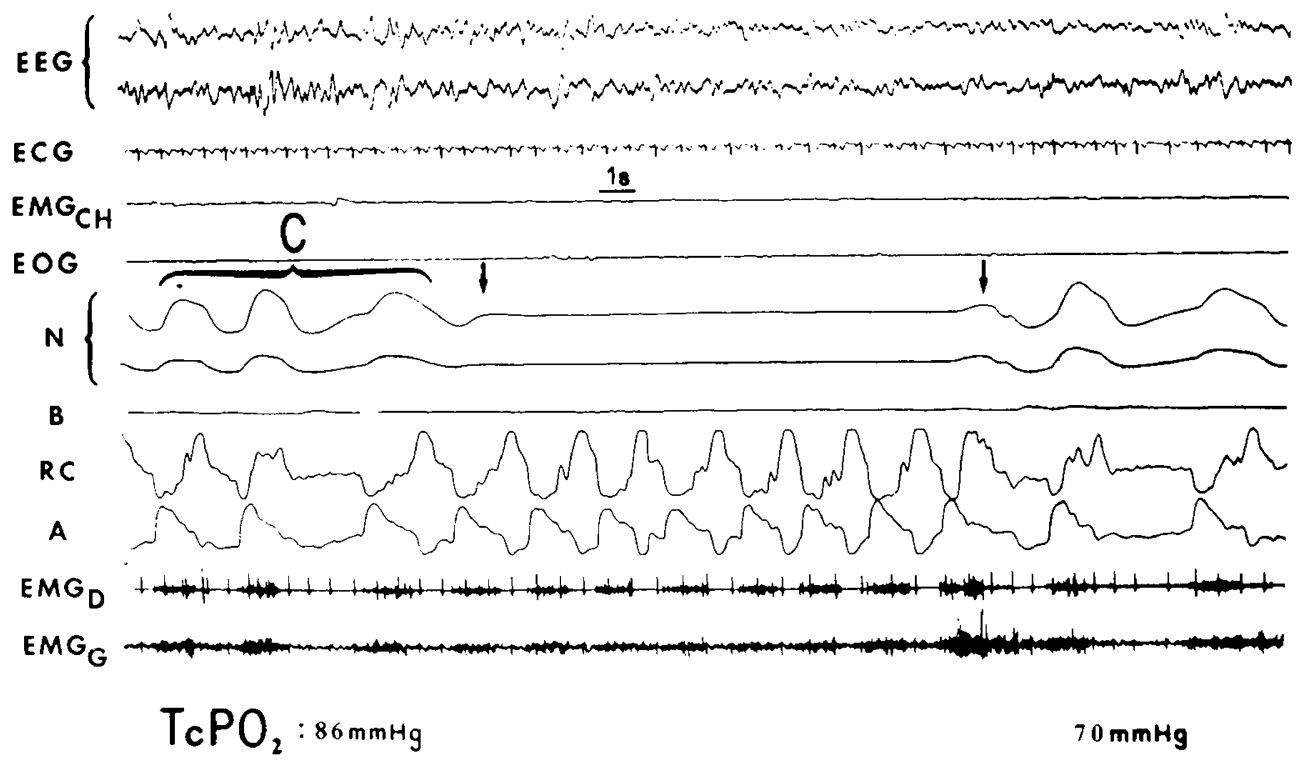

FIG. 1. Representative trace in patient 2. From top to bottom: 1st to 5th traces, electroencephalogram EEG, electrocardiogram (ECG), chin electromyogram $\left(E M G_{C H}\right)$, electrooculogram $(E O G)$; 6 th to 8th traces, nasal $(N)$ and buccal $(B)$ airflow (thermistors); 9 th and 10 th traces, rib cage $(R C)$ and abdominal $(A)$ anteroposterior diameters (magnetometers) with paradoxical inward motion of RC at inspiration; 11 th and 12 th traces, diaphragmatic $(D)$ and genioglossus $(G)$ electromyographic activity $(E M G)$. The arrows show the onset and the end of the apnea; $C$ is the control period, i.e. the three preceding unoccluded breaths. $\mathrm{TcPO}_{2}$ values, before and after the obstructive apnea, are indicated at the bottom of Figure 1.

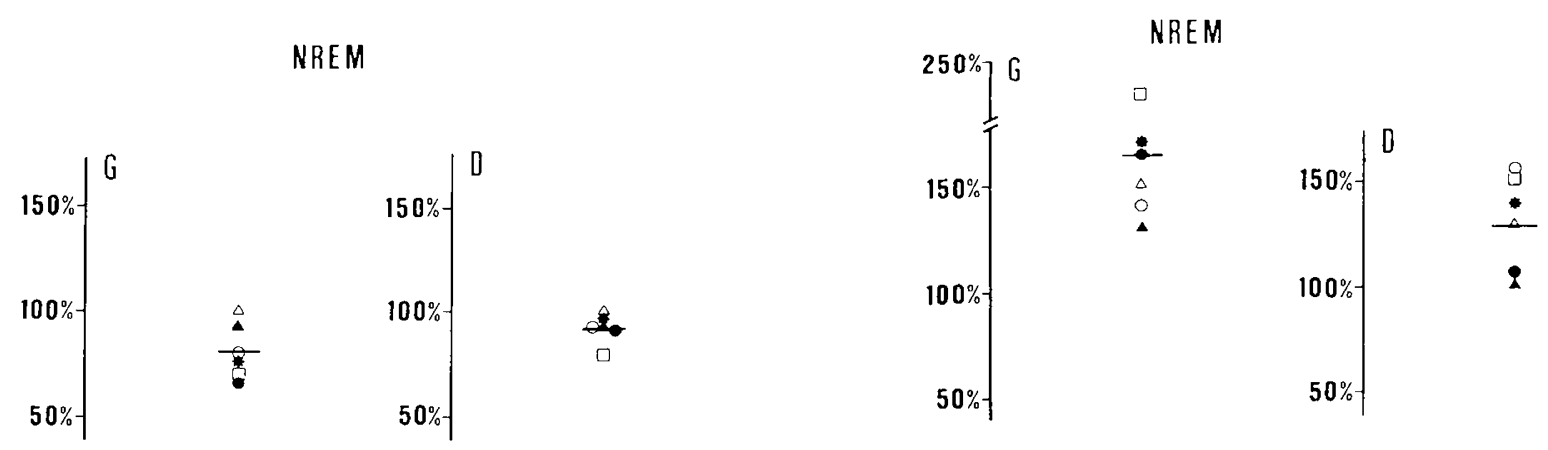

REM

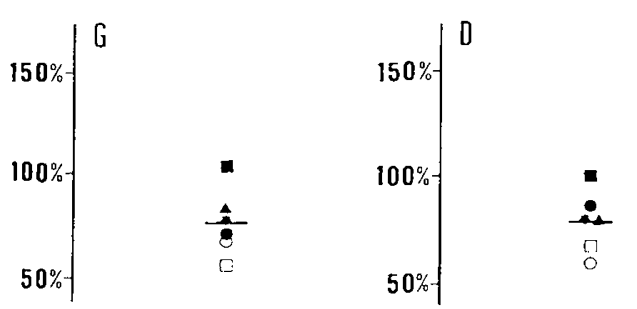

Fig. 2. Diaphragmatic $(D)$ and genioglossal $(G)$ electromyographic activity $(E M G)$ at the onset of obstructive apnea during NREM sleep (upper panel) and REM sleep (lower panel), expressed as a percentage of EMG for control unoccluded breaths $(100 \%)$. Each symbol represents the mean value for the apneas studied in each patient (see Table 1). Bars are the mean value for the whole population in each sleep stage. When compared to control unoccluded breaths, G and D EMG did not significantly change.

Previous reports in infants and children with OSAS have shown that genioglossus EMG activity recorded by surface electrodes was present during sleep $(4,5)$ whereas this activity was not observed in children without OSAS (4). However, in these two studies no attention was paid to either the changes in

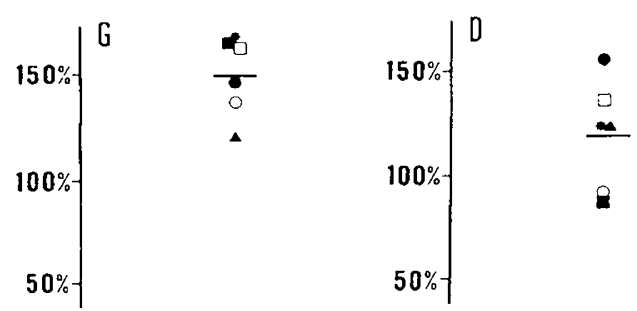

Fig. 3. Diaphragm $(D)$ and genioglossus $(G)$ electromyographic activity $(E M G)$ at the end of obstructive apnea (see Fig. 2 for explanations). Notice the greater increase in G than in D EMG and the decrease in D EMG during REM sleep in patients 5 and 6 (see Table 1 for symbols).

genioglossus EMG activity during the course of obstructive apnea or to the comparison between diaphragmatic and genioglossus EMG activity. We thus focused our study on diaphragmatic and genioglossus EMG activity at the onset and the end of obstructive apnea to see whether or not the same proposed mechanisms for the pathogenesis of obstructive apnea in obese adults with OSAS $(1-3)$ can be applied to children with OSAS. Furthermore, we tested children during natural sleep without preceding sleep 
deprivation or sedation, since both chloral hydrate (6) and sleep deprivation (7) have been shown to depress genioglossus EMG activity.

Onset of apnea. Studies in obese adults with sleep obstructive apneas have suggested two different hypotheses for the onset of obstructive apnea. 1) Onset of obstructive apnea is due to abnormal function of genioglossus and other upper airways dilating muscles, which then fail to counteract the collapsing action of inspiratory muscles $(1,2)$. 2) Onset of obstructive apnea occurs when electrical activity of diaphragm and genioglossus is the lowest, indicating a periodic decrease in neural central drive to both groups of muscles (3). In children with OSAS, our results do not seem to agree with either of the hypotheses formulated in obese adults. Indeed, at the onset of obstructive apnea, we found neither abnormal decrease in genioglossus with persistent diaphragmatic activity, nor significant decrease in both diaphragmatic and genioglossus activity. Obstructive apneas in children could rather be due to sudden increase in upper airway resistance leading to occlusion. This is in agreement with fiberoptic (16) and fluoroscopic $(17,18)$ studies in children with enlarged tonsils which demonstrated that movement of tonsils and pharyngeal walls at inspiration tended to narrow the upper airways and occasionally led to occlusion. Since the tongue was not seen to move backward (17), this suggests that the protruding action of genioglossus was not diminished. It is also possible that in such children upper airway resistance may be periodically also enhanced by fluctuations in the balance between upper airway muscle and diaphragmatic EMG activity (19). In our study we did not find any significant difference between diaphragmatic and genioglossus EMG activity at the onset of the obstructive apnea. However, we did not record other upper airway dilating muscles that may have a decreased activity at the onset of apnea (2).

End of apnea. Previous studies of genioglossus and diaphragmatic EMG in obese adults with OSAS agree that a preferential activation of upper airway dilating muscles compared to the diaphragm is responsible for the resolution of obstructive apnea $(1,3)$. Our results in children with obstructive apnea agree with preferential activation of the genioglossus for resolving obstructive apnea. The current concept of this greater increase in genioglossus activity involves several mechanisms: arousal (20), chemical stimuli (1), and/or reflexes originating from upper airways (3). Arousal does not always precede resolution of obstructive apnea and thus cannot be considered the only causal mechanism (20). The role of chemical stimuli has been emphasized in anesthetized and tracheostomized animals where a preferential rise in genioglossus activity in response to hypoxia and hypercapnia has been demonstrated (21), but its importance is still unclear in humans (22). Moreover, during sleep in obese adults with OSAS, genioglossus activity increases out of proportion with the decrease in arterial oxygen saturation of hemoglobin after the obstructive apneas (3). Our results in children are in agreement with those in adults, since we found no significant relationship between increase in genioglossus EMG activity and decrease in $\mathrm{TcPO}_{2}$. Thus chemical stimuli do not seem to be the sole mechanism involved. Consequently, reflexes originating from upper airways may be implicated in the specific activation of genioglossus activity for the resolution of obstructive apnea (23). The importance of reflexes originating from such receptors has been convincingly pointed out by Onal et al. (3) in obese adults with obstructive apneas where arousal and chemical stimuli did not seem to account for resolution of apnea. They can be similarly implicated in children at the end of obstructive apnea.

Finally, as concerns the diaphragm, diaphragmatic EMG activity increased at the end of obstructive apnea, as previously reported in adults $(3,24)$ except in two children during REM sleep where it decreased. This decrease in diaphragm EMG could be related to reflex activity originating from upper airway receptors and generating diaphragm inhibition such as has recently been described during artificially occluded upper airways in tracheostomized infants (25). We suggest such a reflex might account for inhibition of the diaphragm during resolution of spontaneous obstructive apnea in some children.

\section{CONCLUSION}

Herein we suggest that the mechanisms involved in onset and resolution of sleep obstructive apnea do not seem to be the same in obese adults and in children with obstructive sleep apneas. These differences may be due to 1) different etiologies, i.e. essentially enlarged tonsils in children, 2) duration of symptoms at the moment of the study which is presumably longer in adults than in children, or 3) maturational changes in response to the different stimuli created by upper airway occlusion.

Acknowledgments. The authors thank N. Baala, C. Lebretonnic, and A. Virassamy for their helpful technical assistance and V. Dael and S. Rouchaville for typing the manuscript.

\section{REFERENCES}

1. Remmers JE, Degroot WJ, Sauerland EK, Anch AM 1978 Pathogenesis of upper airway occlusion during sleep. J Appl Physiol 44:931-938

2. Guilleminault C, Hill MV, Simmons FB, Dement WC 1978 Obstructive sleep apnea: electromyographic and fiberoptic studies. Exp Neurol 62:48-67

3. Onal E, Lopata M, O'Connor T 1982 Pathogenesis of apneas in hypersomniasleep apnea syndrome. Am Rev Respir Dis 125:167-174

4. Jeffries B, Brouillette RT, Hunt CE 1984 Electromyographic study of some accessory muscles of respiration in children with obstructive sleep apnea. Am Rev Respir Dis 129:696-702

5. Roberts JL, Reed WR, Mathew OP, Thach BT 1986 Control of respiratory activity of the genioglossus muscle in micrognathic infants. J Appl Physiol 61:1523-1533

6. Hershenson M, Brouillette RT, Olsen E, Hunt CE 1984 The effect of chloral hydrate on genioglossus and diaphragmatic activity. Pediatr Res 18:516-519

7. Leiter JC, Knuth SL, Bartlett JR 1985 The effect of sleep deprivation on activity of the genioglossus muscle. Am Rev Respir Dis 132:1242-1245

8. Rechtschaffen A, Kales A 1971 A manual of standardized terminology, techniques and scoring systems for sleep stages of human subjects. BIS/BRI, ICLA, Los Angeles, CA

9. Prechtl HF, Van Eykern LA, O'Brien MJ 1977 Respiratory muscle EMG in newborns: a non-intrusive method. Early Hum Dev xx:265-283

10. Muller N, Gulston G, Whitton CJ, Froese AB, Bryan MH, Bryan AC 1979 Diaphragmatic muscle fatigue in the newborn. J Appl Physiol 46:688-695

11. Nugent ST, Finley JP 1985 Spectral analysis of the EMG and diaphragmatic muscle fatigue during periodic breathing in infants. J Appl Physiol 58:830833

12. Carlo WA, Miller J, Martin RJ 1985 Differential response of respiratory muscles to airway occlusion in infants. J Appl Physiol 59:847-852

13. Sauerland EK, Sauerland BAT, Orr WC, Hairston LE 1981 Noninvasive electromyography of human genioglossal (tongue) activity. Electromyogr Clin Neurophysiol 21:279-286

14. Guilleminault C, Ariagno R, Korobkin R, Nagel L, Baldwin R, Coons S, Owen M 1979 Mixed and obstructive sleep apnea and near-miss for sudden infant death syndrome. 2. Comparison of near-miss and normal control infants by age. Pediatrics 64:882-891

15. Armitage P 1970 Distribution free-methods. In: Armitage P (ed) Statistical Methods in Medical Research. Blackwell Scientific Publications, London, pp 394-406.

16. Leland L Fan 1984 Transnasal fiberoptic endoscopy in children with obstructive apnea. Crit Care Med 12:590-591

17. Fernach SK, Brouillette RT, Riggs TW, Hunt CE 1983 Radiologic evaluation of adenoids and tonsils in children with obstructive sleep apnea: plain films and fluoroscopy. Pediatr Radiol 13:258-265

18. Felman AH, Loughlin GM, Leftridge CA, Cassisi NJ 1979 Upper airway obstruction during sleep in children. Am J Radiol 133:213-216

19. Hudgel W, Chapman KR, Faulsk C, Hendricks C 1987 Changes in inspiratory muscle electrical activity and upper airway resistance during periodic breathing induced by hypoxia during sleep. Am Rev Respir Dis 135:899-906

20. Kreiger J, Kurtz D 1978 EEG changes before and after apneas. In: Guilleminault C, Dement WC (eds) Sleep Apnea Syndromes. Alan R Liss, New york, pp $161-176$

21. Brouillette RT, Thach BT 1980 Control of genioglossus muscle inspiratory activity. J Appl Physiol 49:801-808

22. Onal E, Lopata M, O'Connor T 1981 Diaphragmatic and genioglossal electromyogram responses to $\mathrm{CO}_{2}$ rebreathing in humans. J Appl Physiol 50:10521055

23. Mathew OP, Abu-Osba YK. Thach BT 1982 Influence of upper airway pressure changes on genioglossus muscle respiratory activity. J Appl Physiol 52:438444

24. Vincken W, Guilleminault C, Silvestri L, Cosio M, Grassino A 1987 Inspiratory muscle activity as a trigger causing the airways to open in obstructive sleep apnea. Am Rev Respir Dis 135:372-377

25. Thach BT, Menon PA, Schefft G 1985 Negative upper airway pressure decreases inspiratory airflow and tidal volume in tracheostomized sleeping human infants. Am Rev Respir Dis 131:295(abstr) 